\title{
Estrategias proyectuales en la enseñanza del proyecto arquitectónico en la Facultad de Arquitectura - UNCP 2018
}

\author{
Project strategies in the teaching of the architectural \\ project in the Faculty of Architecture - UNCP 2018
}

\author{
asanta María Chimbor, C.; Valverde Chávez, M. \\ Facultad de Arquitectura / Universidad Nacional del Centro del Perú \\ Email: csantamaria@uncp.edu.pe
}

\section{Resumen}

La investigación, tiene como propósito conocer las estrategias proyectuales que utilizan los docentes en sus procesos de enseñanza aprendizaje del proyecto arquitectónico en los talleres de diseño de la Facultad de Arquitectura de la UNCP 2018. Este estudio, permitirá a la FARQ/UNCP, contar con la información necesaria para mejorar los procesos de enseñanza aprendizaje del proyecto arquitectónico, retroalimentar el currículo, así como fortalecer la formación de profesionales capaces de asimilar las transformaciones del entorno y responder de manera propositiva e innovadora. El tipo de investigación es aplicada de nivel descriptivo, con un diseño no experimental, transversal y censal; es decir, se trabajó con 30 docentes coordinadores que enseñan los talleres de diseño arquitectónico.

Se utilizó una encuesta para los docentes, validada por expertos. Estadísticamente se utilizaron las frecuencias, las mismas que fueron procesadas a través de tablas y gráficos con el SPSS versión 22. Los resultados demostraron que solo el 41,67 \% de los docentes, mencionan que implementan estrategias proyectuales generales en la enseñanza del proyecto arquitectónico; en tanto que, una mayoría ( $58.33 \%$ ) deja que el alumno proponga su propia estrategia y, sobre ello, el docente le ayuda a implementarlo. En todo caso, ningún profesor se exime de la utilización de alguna estrategia, en su proceso. Finalmente, se recomienda que la Facultad de Arquitectura de la UNCP, implemente ciclos de capacitación docente, en cuanto a utilización de estrategias proyectuales, pues se tiene un déficit cuantitativo y cualitativo de ello.

Palabras clave: estrategias proyectuales, proyecto arquitectónico, enseñanza-aprendizaje, capacitación docente

\begin{abstract}
The purpose of the research is to know the project strategies used by teachers in their teaching-learning processes of the architectural project in the design workshops of the Faculty of Architecture of the UNCP 2018. This study will allow the FARQ / UNCP to count on the necessary information to improve the teaching-learning processes of the architectural project, provide feedback to the curriculum, as well as strengthen the training of professionals capable of assimilating the transformations of the environment and respond in a proactive and innovative manner. A quantitative - qualitative, descriptive, and censal study was carried out, in other words, we worked with all the teachers that teach the architectural design workshops, that is, 30 teachers.

An expert-validated teacher survey was used. The frecuencies that were processed through tables and graphs with the SPSS version 22. The results showed that only $41.67 \%$ of the teachers say that they implement general project strategies in the teaching of the architectural project, while a majority $(58.33 \%$ ) allows the student to propose their own strategy and about it the teacher helps him to implement it. In any case, no teacher is exempt from the use of a strategy in his process. Finally, it is recommended that the Faculty of Architecture of the UNCP, implement teacher training cycles in terms of the use of project strategies, because we have a quantitative and qualitative deficit Keywords: Strategies, design, project, workshops.
\end{abstract}

Keywords: project strategies, architectural project, teaching-learning, teacher training 


\section{Introducción}

En el campo de la enseñanza de la arquitectura, y en particular del proyecto arquitectónico, la selección y utilización de estrategias en cada una de las etapas que conciernen al desarrollo del proyecto arquitectónico, se convierte como el punto medular de este proceso. No en vano, hoy los arquitectos contemporáneos están más preocupados en desarrollar estrategias operacionales que lo lleven con éxito a configurar un proyecto arquitectónico.

En la enseñanza de la arquitectura, la actividad preponderante a realizar en el aula es el diseñar, esta actividad se convierte en el elemento determinante o neurálgico de la Carrera de Arquitectura, a través de lo que se denomina como el "proyecto arquitectónico”, en la asignatura denominada, ya sea, Taller de Diseño Arquitectónico, Taller de Arquitectura o Taller de Proyectación Arquitectónica. En estos talleres, confluyen las otras cuatro áreas de la misma, es aquí donde se observa y se aplica la Historia de la Arquitectura, el Urbanismo, las Estructuras, las Tecnologías de la Construcción, los Dibujos y Representaciones y, es por ello, que radica la importancia de valorar y evaluar los actuales métodos de enseñanza en el Taller de Diseño Arquitectónico.

En los cursos de diseño o proyectos, es donde se presentan los mayores problemas del proceso de enseñanza. En muchas facultades de Arquitectura, los maestros improvisan, no preparan su clase y ni siquiera su curso cuenta con un programa. Así, las asignaturas de diseño o proyectos se caracterizan por la falta de una metodología (solo se plantea el "dale vueltas") y en ellos no se le enseña al alumno a proyectar, solo se ve la inspiración espontánea (muchas veces orientado únicamente el gusto del profesor y los aspectos formales en moda); sin existir, por parte del maestro, un análisis crítico de los proyectos de sus alumnos, donde se den las razones objetivas de los aciertos o desaciertos en los mismos. Lo anterior implica que muchos profesores ven el diseño únicamente como un acto de "libre inspiración".

En los casos más alentadores, el docente puede explicar el proceso de diseño, identificando cada una de sus partes; es decir, el contenido. Pero cuando estos contenidos deben ser aplicados a la especificidad de un proyecto arquitectónico, se nota una clara ausencia de estrategias proyectuales que permitan la construcción de cada componente del diseño, ya sea en la fase de la investigación (conceptualización) o la de realización proyectual.

En síntesis, podemos citar como referencia tres autores, de los cuales el lector puede extraer las estrategias operativas que utilizan los arquitectos contemporáneos representativos.
Moneo (2004), en su publicación "Inquietud teórica y estrategia proyectual en la obra de ocho arquitectos contemporáneos", describe las estrategias proyectuales de arquitectos contemporáneos importantes, como Stirling, Venturi, Rossi, Ensenan, Siza, Gehry, Koolhaas y Herzog \& de Meuron; en esa obra, Moneo (2004) explica que el término "estrategia" se entienden como mecanismos, procedimientos, paradigmas y artilugios que aparecen con recurrente insistencia en la obra de estos arquitectos y que entiende que ellos se valen para configurar lo construido.

La habilidad del manejo de la sección y las plantas como herramienta fundamental en la obra de Stirling, la fragmentación de formas a través de la manipulación de maquetas, la isometría y el diagrama como herramientas de diseño en Eisenmman y Rossi y; su estrategia proyectual de la tipología (memoria construida de los pueblos), Rem Kolhaas y sus estrategias relacionadas con el vacio, la gravedad y el montaje, permiten al lector identificar formas y modos operativos de cómo estos arquitectos traducen sus ideas en formas arquitectónicas.

El segundo autor de vital importancia es Eisenman (2015), quien en su libro "Diez edificios canónicos 1950-2000" analiza las obras de arquitectos comprendidos en el periodo de 1950 al año 2000, seleccionadas en su carácter de proyectos bisagra en el discurso arquitectónico; en cada caso, señalan un cambio, una ruptura con los discursos arquitectónicos vigentes en su momento.

Además, se puede distinguir algunas estrategias utilizadas por los arquitectos seleccionados; el uso del diagrama de paraguas en Mies Van Der Rohe, las herejías textuales usadas por Le Corbusier en el Palacio de Congresos de Estrasburgo, el espacio diacrónico de Kahn, el diagrama de los nueve cuadrados utilizado por Venturi en la Casa Vanna Venturi, estrategias de textos de analogía utilizados en el Cementerio de San Cataldo por Aldo Rossi, las estrategias del vacío de Koolhaas, la deconstrucción del eje usado por Daniel Libeskind, en Museo Judío de Berlín y el diagrama del paraguas blando utilizado por Gehry en el Edifico Peter B. Lewis. Todas estas estrategias pueden servir como referentes importantes en los procesos de formación de los estudiantes que lo sitúen en este momento contemporáneo.

Finalmente, García (2012), en "Estrategias operativas en arquitectura - Técnicas de proyecto de Price a Koolhas”, analiza algunas estrategias contemporáneas, como el uso del "tercer paisaje fragmentado" aplicado por el francés Gilles Clement, las estrategias programáticas en torno a la densidad utilizadas por Koolhas en el proyecto para la rehabilitación de la Central Térmica de Battersea en Londres y su transformación en el Museo Tate Modern; la recuperación del "es- 
pacio genérico" y construcción de relaciones usada como estrategia proyectual por Foster en el edificio Fred Olsen Amenity Centre de 1970 y; una propuesta que llama la atención, es la referida a una estrategia opuesta al que tradicionalmente fue el cometido de la disciplina, y que crece en importancia "hacer casi nada”. La observación atenta, la reconsideración de lo existente, la coexistencia, la modestia, el trabajo por substracción más que por adición, la acupuntura, la no intervención o directamente la demolición se perfilan como vías futuras para entender el ejercicio profesional.

El objetivo de la presente investigación, fue la determinación del tipo de estrategias proyectuales que utilizan los docentes en la enseñanza del proyecto arquitectónico en los talleres de diseño de la UNCP $-2018$.

\section{Métodos y materiales}

El tipo de investigación fue aplicada, según Carrasco (2009), esta investigación se caracteriza porque sus resultados son aplicados de inmediato. El nivel de investigación fue descriptivo, como afirman Hernández et al., (1991), por centrarse en identificar las características cuantitativas o cualitativas de la variable de estudio. El diseño fue no experimental transversal y descriptivo.

La población, estuvo formado por los docentes coordinadores de la Facultad de Arquitectura de la Universidad Nacional del Centro del Perú, que enseñan el Taller de Diseño Arquitectónico.

La recopilación de datos, se hizo a través de una encuesta semi estructura y con preguntas libres. Esta encuesta contó con 18 ítems con preguntas abiertas. Este cuestionario fue validado por la opinión de tres expertos.

El procesamiento y análisis de los datos, se realizó con el programa estadístico Exel, en donde se análisó a través de estadísticos descriptivos, iniciándose el trabajo con reportes de frecuencias simples y acumulados tanto absolutos como relativos para la determinación de parámetros, siendo la media moda y mediana.

Los resultados fueron presentados en tablas de distribución, frecuencias, con porcentajes respectivos y en gráficos de barra. Los resultados responden a entrevistas hechas a los docentes coordinadores de los talleres de diseño del periodo lectivo 2018-I de la Facultad de Arquitectura de la UNCP, que en total fueron 12.

\section{Resultados}

Del total de docentes entrevistados, solo el 41,67 $\%$ dicen que implementa estrategias proyectuales generales en la enseñanza del proyecto arquitectónico; en tanto que, la mayoría $(58,33 \%)$ menciona que deja que el alumno proponga su propia estrategia y, sobre ello, el docente le ayuda a implementarlo. La distribución de estrategias se muestra en la Figura 1.

\section{Figura 1}

Estrategias generales aplicados en la enseñanza de arquitectura. Donde: (1) es pasar de lo intuitivo a lo racional, (2) Ir de lo abstracto a lo concreto, (3) plantear un argumento y plasmarlo en una forma y (4) uso de imágenes.

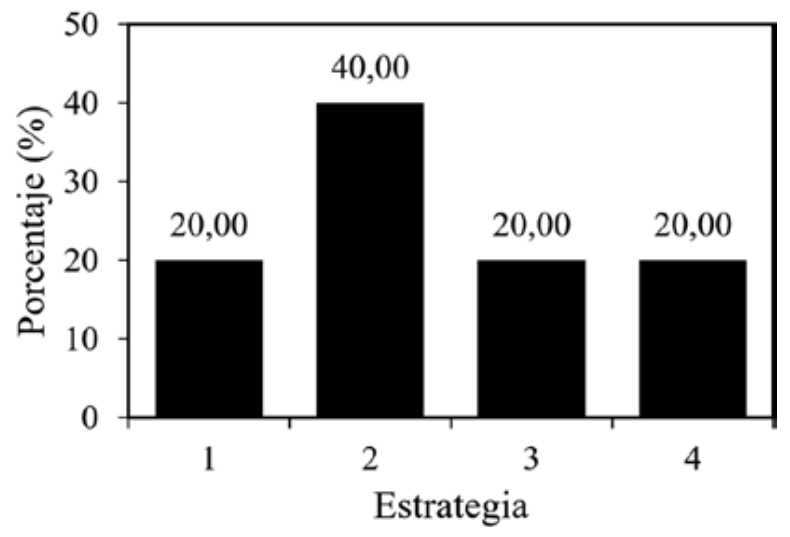

Además, se observó que un 66,67 \% de docentes implementó estrategias proyectuales para enfrentar el trabajo de planteamiento del problema, en tanto que $33,23 \%$ de docentes deja que el alumno establezca su propia estrategia. En esta etapa no se trabajó sin implementar estrategias.

De los docentes que manifestaron que, sí implementaban estrategias proyectuales en la fase de planteamiento del problema en la enseñanza del proyecto arquitectónico, se tiene que el análisis FODA y la estrategia de utilizar el "Árbol de problemas" alcanzaron el mayor porcentaje (25\%); en tanto que, el resto de estrategias planteadas alcanzan un 12,5\%. En la Figura 2, se muestra el tipo de estrategias utilizadas en esta fase del planteamiento del problema.

\section{Figura 2}

Tipo de estrategias utilizadas en la fase del planteamiento del problema en la enseñanza del proyecto arquitectónico. Donde: (1) análisis FODA, (2) árbol de problema, (3) matriz de valoracion de problemas, (4) mapeos, (5) revisión bibliográfica y (6) indeed values.

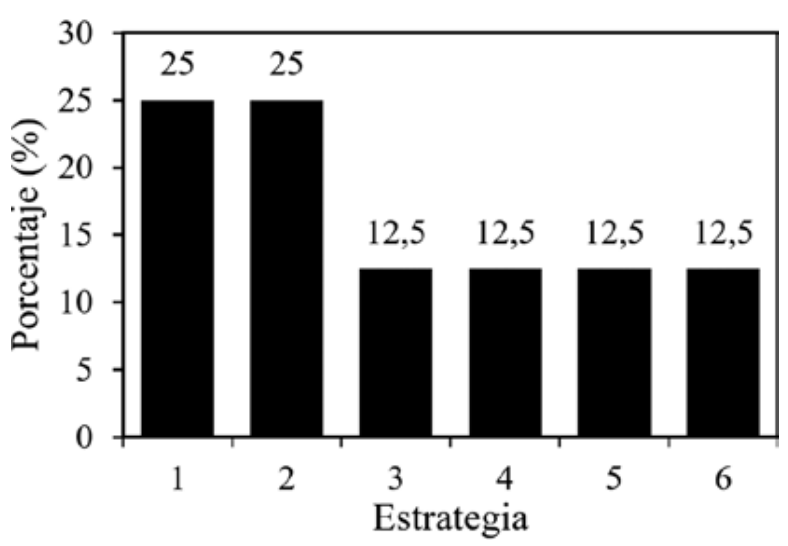


Se observó que un 66,67 \% de docentes implementa estrategias proyectuales para enfrentar el trabajo de análisis del contexto, en tanto que 33,23\% de docentes deja que el alumno establezca su propia estrategia. En esta etapa no se trabaja sin implementar estrategias.

De los docentes que manifestaron que, si implementaban estrategias proyectuales en la fase de análisis del contexto en la enseñanza del proyecto arquitectónico, se tiene la estrategia basada en esquemas gráficos (lugar-usuario-objeto), es la más frecuente (37,5\%), luego está el uso de diagramas del contexto con un 25 $\%$; en tanto que, las estrategias referidas a uso de mapas temáticos, investigación proyectual y análisis FODA, están en tercer lugar con un 12,5\% cada una. Ello se observa en la Figura 3.

\section{Figura 3}

Tipo de estrategias para el análisis del contexto en la enseñanza del proyecto arquitectónico. Dónde: (1) Uso de mapas temáticos, (2) Diagramas abstractos del contexto, (3) Esquemas gráficos (lugar-usuario-objeto), (4) Investigación proyectual y (5) Análisis FODA

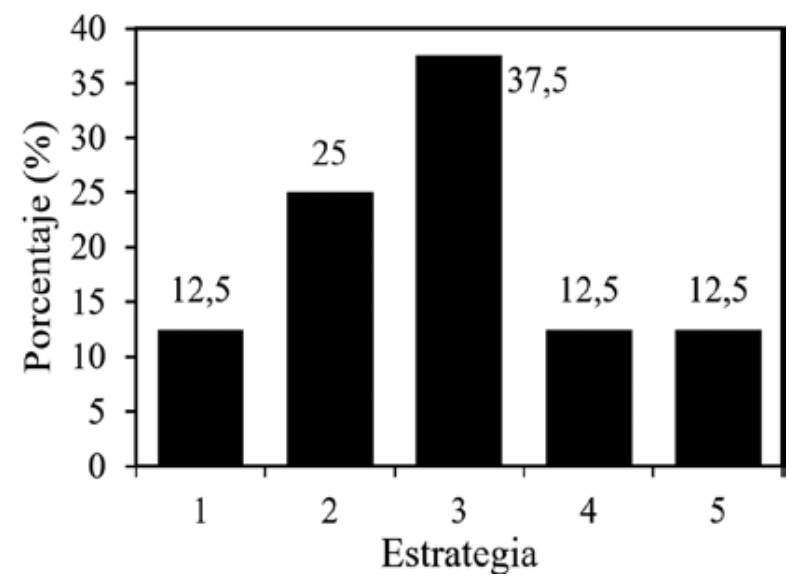

Por otra parte, se observó que un $75 \%$ de docentes implementa estrategias proyectuales para enfrentar la fase de ideación en la enseñanza del proyecto arquitectónico, en tanto que un $25 \%$ de docentes deja que el alumno establezca su propia estrategia. En esta etapa no se trabaja sin implementar estrategias.

De los docentes que manifestaron que, si implementaban estrategias proyectuales en la fase de ideación en la enseñanza del proyecto arquitectónico, se tiene que la estrategia basada en la definición del concepto arquitectónico, es la más frecuente (44,44\%), luego está la definición de la idea rectora y la de establecer las fuerzas del lugar con un 22,22\%; en tanto que, la estrategia referida a la definición del programa arquitectónico está en tercer lugar, con un 11,11\%. Ello se observa en la Figura 4.

\section{Figura 4}

Tipo de estrategias utilizadas en la fase de ideación en la enseñanza del proyecto arquitectónico. Donde: (1) Definir idea rectora, (2) definición del concepto arquitectónico, (3) definir el programa arquitectónico y (4) establecer las fuerzas del lugar.

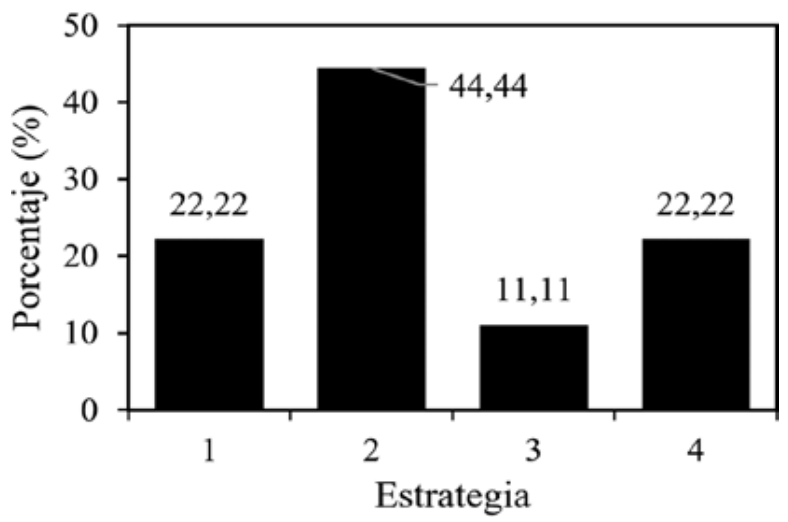

Adicionalmente, el 83,33\% de docentes implementaron estrategias proyectuales para enfrentar la fase de transformación de las ideas a las formas en la enseñanza del proyecto arquitectónico, en tanto que un 16,67 \% de docentes deja que el alumno establezca su propia estrategia.

De los docentes manifestaron que, si implementaban estrategias proyectuales en la fase de transformación de las ideas a las formas en la enseñanza del proyecto arquitectónico, se tiene que la estrategia basada en el partido arquitectónico es la más frecuente (40\%), luego está el uso de analogías y metáforas y el plan de masas con un $20 \%$ cada una; en tanto que, las estrategias referidas al brainstormig y la gestación creativa en tercer lugar con un $10 \%$ cada una. Ello se muestra en la Figura 5.

\section{Figura 5}

Tipo de estrategias utilizadas en la fase de transformación de las ideas a las formas en la enseñanza del proyecto arquitectónico. Dónde: (1) Brainstorming, (2) Partido arquitectónico, (3) Uso de analogías y metáforas, (4) Plan de masas y (5) Gestación creativa.

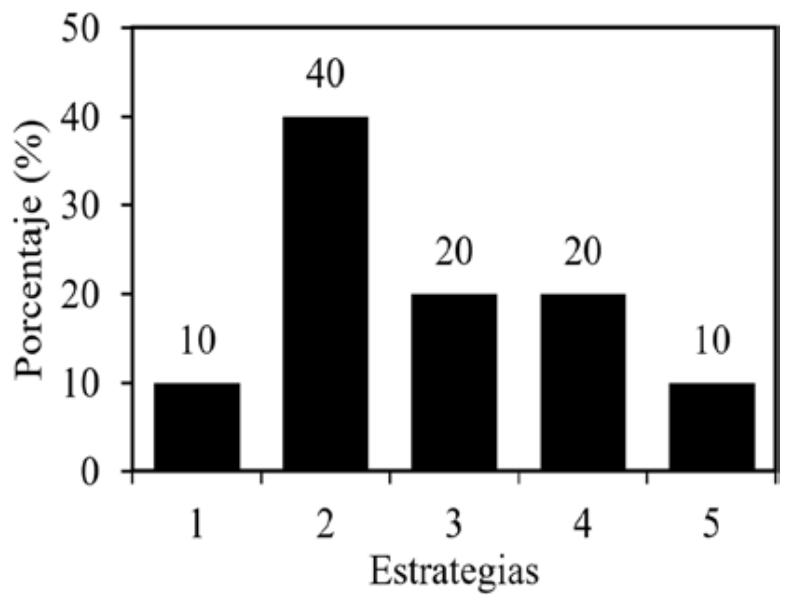

Se observó que un 58,3\% de docentes deja que el alumno establezca su propia estrategia en el desarrollo del sistema arquitectónico de la función, en tanto que el 41,7 \% implementa estrategias proyectuales para enfrentar esta fase. 
De los docentes que manifestaron que, sí implementaban estrategias proyectuales en la fase de desarrollo del sistema forma-función, referidos a la función en la enseñanza del proyecto arquitectónico, se tiene que la estrategia basada en los diagramas funcionales es la más frecuente (40\%); en tanto que, las estrategias referidas a la zonificación, estudio de referentes funcionales y uso del programa arquitectónico con un 20 \% cada una. Lo mismo se observa en la Figura 6.

\section{Figura 6}

Tipo de estrategias utilizadas en la fase de desarrollo del sistema forma - función en la enseñanza del proyecto arquitectónico. Dónde: (1) Zonificación, (2) Diagramas funcionales, (3) Estudio de referentes funcionales y (4) Uso del programa arquitectónico.

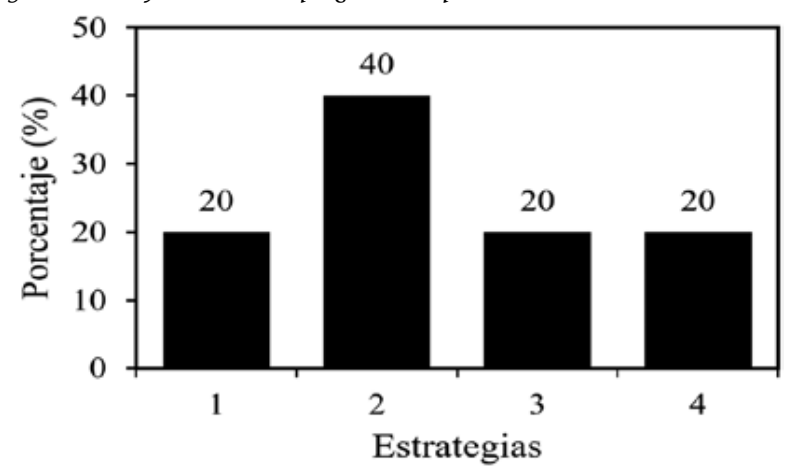

Finalmente, se determinó que un 58,3 \% de docentes deja que el alumno establezca su propia estrategia en el desarrollo del sistema arquitectónico de la forma; en tanto que, el 41,7\% implementa estrategias proyectuales para enfrentar esta fase.

De los docentes que manifestaron que, sí implementaban estrategias proyectuales en la fase de desarrollo del sistema forma-función, referidos a la forma en la enseñanza del proyecto arquitectónico, se tiene que la estrategia basada en modelos referentes (maquetas y 3D) es la más frecuente (50\%); en tanto que, las estrategias referidas a la geometrización del proyecto, calca sucesiva y el uso de software para modelación de la forma tienen un $20 \%$ cada una. Ello se muestra en la Figura 7.

\section{Figura 7}

Tipo de estrategias utilizadas en la fase de desarrollo del sistema forma-función (forma) en la enseñanza del proyecto arquitectónico. Dónde: (1) Geometrización del proyecto, (2) Uso de modelos referentes (maquetas y 3D), (3) Calca sucesiva y (4) Uso de software para modelación de la forma.

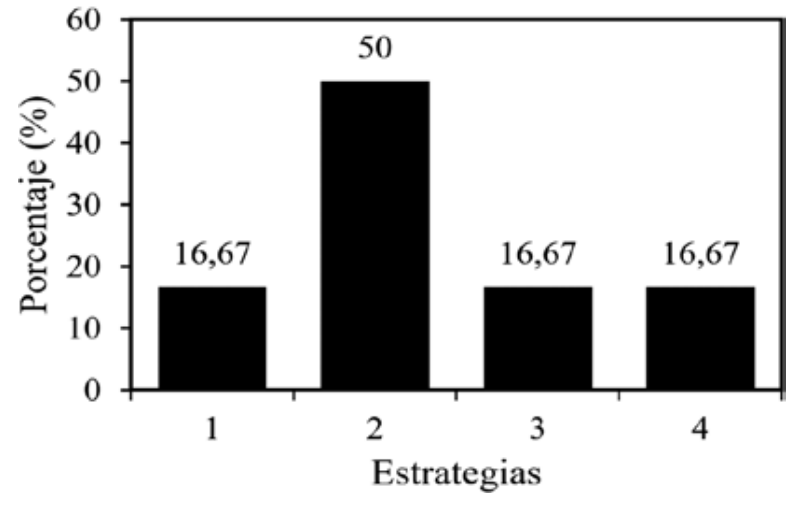

\section{Resultados}

Un primer aspecto, que se aprecia a partir de los resultados es que, si bien es cierto la mayoría de los arquitectos docentes que enseñan proyectos arquitectónicos en los talleres de diseño de la Facultad de Arquitectura de la UNCP, muestran una conformidad a las fases del proyecto arquitectónico sugerido, es evidente que la mayoría no cuenta con una sistemática racional en cuanto a su proceso de diseño. La dispersión de opiniones, que en muchos casos no tienen que ver con fases, sino con acciones puntuales, así lo demuestra. Sugerencias como; fase de zonificación, de ideación donde se desarrollan las formas o secuencia no lineal al proceso propuesto, ponen en evidencia la falta de análisis y de coherencia en sus sugerencias.

Entendiendo, en términos generales, que una estrategia viene a ser una forma o un modo de enfrentar un problema específico, en este caso dentro del proceso de diseño, un porcentaje relativamente bajo de docentes implementa una serie de estrategias proyectuales generales $(41.57 \%)$, dejando que los alumnos elaboren sus propias estrategias en un $50.33 \%$, ayudándoles sobre esa base a desarrollar las mismas. Estos resultados no son sorprendentes, pues la forma tradicional de la enseñanza del proyecto arquitectónico, que todavía tiene cabida en universidades como la nuestra, hace que el docente no prepare una clase, sino que se respalde solamente sobre la crítica del proyecto que solo implica en informarle al alumno que aspectos de su proyecto no están bien elaborados, de acuerdo a su opinión.

Pero también, hay que remarcar que ese porcentaje de docentes que implementan estrategias proyectuales en su proceso de enseñanza del proyecto arquitectónico, implementan estrategias demasiado convencionales y tradicionales, que vienen desde su formación profesional y que, pese al transcurrir de los años y la evolución que viene teniendo la arquitectura, no han renovado las mismas. Las estrategias señaladas en el marco teórico, que corresponde a la arquitectura contemporánea, no son abordadas, ni siquiera mencionadas por los docentes, ni a nivel general ni en las fases específicas. Estrategias como el pliegue y despliegue (Chiarela, 2001) y espacios regenerativos o la estrategia del vacío (García, 2012), no han sido mencionados por los docentes de los talleres de diseño de la Facultad de Arquitectura de la UNCP. La mayoría manifiesta que la estrategia proyectual que utiliza fue aprendida en la universidad, pero que en el camino y que a lo largo de su quehacer arquitectónico fueron influenciados por la experiencia, algo así como autodidactas.

Se puede afirmar que, los arquitectos encuestados, aún no han renovado sus formas de diseño, ya que sus estrategias todavía corresponden a las aprendidas en la universidad y que no están a tono de las estrategias que se desarrollan en el mundo contemporáneo. 
Aún no se tiene una idea clara de verdadero significado de lo que es una "estrategia proyectual", pues las respuestas fueron múltiples y en la mayoría de casos ambiguas. El marco teórico desarrollado al respecto, pone en evidencia la falta de claridad de los arquitectos entrevistados en torno al tema.

Se puede observar en los resultados, que son las etapas correspondientes al planteamiento del problema y el análisis del contexto donde se encuentra una mayor preocupación por los docentes de implementar estrategias que operativicen estas etapas $(66.67 \%)$. Sin embargo, la estrategias planteadas no son creadas específicamente para el trabajo de diseño arquitectónico, sino ha sido adoptas de otros procesos, como el FODA en la planificación o el árbol de problemas de los proyectos de inversión.

Dentro de las fases de diseño señalado y que un alto porcentaje de docentes está de acuerdo, es la fase de desarrollo del sistema formal-funcional (anteproyecto), donde se encuentra una mayor falencia de implementación de estrategias por parte del docente, dejando que sea el alumno implemente alguna estrategia que luego el apuntala. La carencia de una teoría del proyecto arquitectónico que guíe el proceso genera esta dificultad; por ello, es que generalmente los alumnos no hacen sino copiar las estructuras funcionales y formales de proyectos existentes en las revistas o libros.

\section{Conclusiones}

- La mayoría de docentes, dejan que sean los alumnos que implementen sus propias estrategias proyectuales. La estrategia más utilizada para este efecto es de lo abstracto a lo concreto. Mientras que la mayoría de docentes implementan estrategias proyectuales para enfrentar el trabajo de planteamiento del problema.

- De los docentes que manifestaron que, sí implementaban estrategias proyectuales en la fase de planteamiento del problema en la enseñanza del proyecto arquitectónico, se tiene que el análisis FODA y la estrategia del “Árbol de problemas” alcanzan el mayor porcentaje.

- La mayoría de docentes, implementan estrategias proyectuales para enfrentar el trabajo de análisis del contexto. De los docentes que manifestaron que, si implementaban estrategias proyectuales en la fase de análisis del contexto en la enseñanza del proyecto arquitectónico, se tiene la estrategia basada en esquemas gráficos (lugar-usuario-objeto), es la más frecuente.

- Un $75 \%$ de docentes, implementan estrategias proyectuales para enfrentar la fase de ideación en la enseñanza del proyecto arquitectónico. En la misma línea, se tiene que la estrategia basada en la definición del concepto arquitectónico, es la más frecuente.
- Un alto porcentaje de docentes, implementan estrategias proyectuales para enfrentar la fase de transformación de las ideas a las formas en la enseñanza del proyecto arquitectónico. De los docentes que manifestaron que, si implementaban estrategias proyectuales en la fase de transformación de las ideas a las formas en la enseñanza del proyecto arquitectónico, se tiene que la estrategia basada en el partido arquitectónico, es la más frecuente.

- Poco más de la mitad de docentes, dejan que el alumno establezca su propia estrategia en el desarrollo del sistema arquitectónico de la función. De los docentes que manifestaron que, si implementaban estrategias proyectuales en la fase de desarrollo del sistema forma-función, referidos a la función en la enseñanza del proyecto arquitectónico, se tiene que la estrategia basada en los diagramas funcionales es la más frecuente.

- En la misma línea, la mayor parte de docentes dejan que el alumno establezca su propia estrategia en el desarrollo del sistema arquitectónico de la forma. De los docentes que manifestaron que, sí implementaban estrategias proyectuales en la fase de desarrollo del sistema forma-función, referidos a la forma en la enseñanza del proyecto arquitectónico, se tiene que la estrategia basada en modelos referentes (maquetas y 3D), es la más frecuente.

\section{Referencias bibliográficas}

Aguilar, M. (2000). Camino al diseño - Proceso del diseño arquitectónico. Manizales, Colombia.

Alexander, C. (1976). Ensayo sobre la sintesis de la forma. Buenos Aires.: Infinito.

Aliata, F. (2013). Estrategias proyectuales. Buenos Aires: Nobuko.

Bermúdez, J. (2013). Sobre el partido arquitectónico. Utah, EE.UU

Boix, F. \& Montepare, A. (2012). El Proyecto arquitectónico - enseñanza y práctica de las estrategias proyectuales. Buenos Aires: Universidad Abierta Interamericana.

Camacho, M. (1999). Diccionario de arquitectura y Urbanismo. México: Trillas.

Carrasco, S. (2009). Metodología de la investigación científica. Lima: San marcos.

Chiarela, M. (2001). Pliegues, despliegues y repliegues. Didáctica proyectual e instrumentos de Ideación. Arquitectura revista Vol. 7, n. 1, 63-67.

Corral, G. (2007). El proyecto de arquitectura como una forma de producción del conocimiento; Hacia la investigación proyectural. Revista de Arquitectura. 
Eisenman, P. (2015). Diez edificios canonicos 1950-2000. Barcelona: Gustavo gilli.

Eligio Triana, César Andrés \& Verdugo Reyes, Hernando. (2009). El proyecto clásico en arquitectura. Aproximación a una estrategia. Revista de Arquitectura, 74-82.

García, J. (2012). Estrategias operativas en arquitectura - Técnicas de proyecto de Price a Koolhaas. Buenos Aires: Nobuko.

Hernández, R.; Fernández, C. y Baptista, P. (1991). Metodología de la investigación. D.F. México: Mc Graw Hill Interamericana Editores.

Ludeña, W. (1977). Ideas y arquitectura en el Perú del siglo $X X$. Lima: SEMSA.
Moneo, R. (2004). Inquietud teórica y estrategia proyectual. Barcelona: Actar.

Muñoz, A. (2008). El Proyecto de arquitectura. Barcelona: Reverté.

Piña Lupianez, R. (2004). El proyecto de arquitectura. El rigor científico como instrumento poético. Madrid: Tesis Doctoral.

Saslz, M. (2015). Estrategias proyectuales. Junya Ishigami. Madrid, Madrid.

Terán, J. A. (2005). Reflexiones sobre la enseñanza de la arqutiectura. Seminario de Teoría e Historia. (D. d. UNAM., Ed.) D.F. México., México. 